\title{
On restrained edge dominating set of graphs
}

\author{
S. K. Vaidya ${ }^{1 *}$ and P. D. Ajani ${ }^{2}$
}

\begin{abstract}
Keywords

AMS Subject Classification

05 C69.

${ }^{1}$ Department of Mathematics, Saurashtra University, Rajkot-360005, Gujarat, India.

${ }^{2}$ Department of Mathematics,, Atmiya University, Rajkot - 360005, Gujarat, India.

*Corresponding author: ${ }^{\text {* }}$ samirkvaidya@yahoo.co.in; ${ }^{2}$ paragajani@gmail.com

Article History: Received 11 October 2019; Accepted 27 November 2019
\end{abstract}

For a graph $G=(V, E)$, a subset $D$ of $E$ is restrained edge dominating set of $G$ if every edge not in $D$ is adjacent to an edge in $D$ as well as an edge in $E-D$. The restrained edge domination number of $G$, denoted by $\gamma_{r e}(G)$ is the minimum cardinality of a restrained edge dominating set of $G$. Here, we characterize restrained edge dominating set and also investigate restrained edge domination number of some wheel related graphs.

Dominating set, restrained dominating set, restrained edge dominating set, restrained edge domination number.

(C)2020 MJM.

\section{Contents}

1 Introduction $\ldots \ldots \ldots \ldots \ldots \ldots \ldots \ldots \ldots \ldots \ldots \ldots \ldots, 28$

2 Main Results...............................29

References $\ldots \ldots \ldots \ldots \ldots \ldots \ldots \ldots \ldots \ldots \ldots \ldots \ldots \ldots, \ldots \ldots \ldots$

\section{Introduction}

The domination in graphs is one of the most rapidly growing areas within and out side of graph theory. It has attracted many researchers to work on it due to its diversified applications in the fields like linear algebra and optimization, design and analysis of communication networks.

By a graph $G=(V, E)$, we mean simple, finite, connected and undirected with vertex set $V$ and edge set $E$. An edge $e$ of a graph $G$ is said to be incident with the vertex $v$ if $v$ is an end vertex of $e$. Two vertices $u$ and $v$ of $G$ connected by an edge $e$ are called adjacent vertices. Two edges $e$ and $f$ of $G$ having a vertex $v$ in common are called adjacent edges. An independent edges is the set of edges, in which no two edges are adjacent. The minimum degree among the vertices of graph $G$ is denoted by $\delta(G)$ while the maximum degree among the vertices of graph $G$ is denoted by $\Delta(G)$. For any vertex $v \in V$, the open neighbourhood of $v$ is defined as $N(v)=\{u \in V / u v \in E\}$. The number of elements of a set $A$ is denoted by $|A|$. For any undefined term we refer to Harary [4].
A set $S \subseteq V$ of vertices in a graph $G$ is called a dominating set if every vertex $v \in V$ is either an element of $S$ or is adjacent to an element of $S$. A dominating set $S$ is a minimal dominating set if no proper subset $S^{\prime} \subset S$ is a dominating set. The minimum cardinality of a dominating set of $G$ is called domination number, denoted by $\gamma(G)$ and the corresponding dominating set is called a $\gamma$-set of $G$. A brief account of dominating set and its related concepts can be found in Haynes et al [5].

In a graph $G$, a vetex of degree one is called a pendant vertex and an edge incident with a pendant vertex is called a pendant edge.

An edge analogue of dominating set is also available. A subset $F \subseteq E$ is an edge dominating set if each edge in $E$ is either in $F$ or is adjacent to an edge in $F$. An edge dominating set $F$ is called a minimal edge dominating set if no proper subset $F^{\prime}$ of $F$ is an edge dominating set. The minimum cardinality among all minimal edge dominating sets is called the edge domination number, denoted by $\gamma_{r e}(G)$. Mitchell and Hedetniemi [7] introduced the concept of edge domination. Vaidya and Pandit [13] have discussed edge domination in some path and cycle related graphs. Arumugam and Velammal [1] have studied the edge domination in graphs. The complementary edge domination in graphs is well studied by Kulli and Soner [6] while Yannakakis and Gavril [14] have explored edge dominating sets in graphs. 
Many variants of dominating sets are available in the existing literature. One such variant is restrained dominating set, a set $S \subseteq V$ is a restrained dominating set if every vertex not in $S$ is adjacent to a vertex in $S$ as well as to a vertex in $V-S$. The minimum cardinality of a restrained dominating set $S$ is called the restrained domination number of $G$, denoted by $\gamma_{r}(G)$. The concept of restrained domination was introduced by Telle and Proskurowski [9] as a vertex partitioning problem. Restrained domination in the context of path, cycle and wheel is discussed by Vaidya and Ajani [10-12] while the restrained domination of complete graph, multipartite graphs and the graphs with minimum degree two is well studied by Domke et al $[2,3]$. These variants were introduced by identifying one or more characteristics of elements of vertex subset or edge subset.

In this paper we have discussed an edge analogue of restrained domination in graphs. For a graph $G=(V, E)$, a set $F \subseteq E$ is a restrained edge dominating set if every edge not in $F$ is adjacent to an edge in $F$ and also adjacent to an edge in $E-F$. The minimum cardinality of restrained edge dominating set of $G$ is called restrained edge domination number, denoted as $\gamma_{r e}(G)$. This concept was conceived by Soner and Ghobadi [8].

In the present work we characterize restrained edge dominating set and also investigate restrained edge domination number of some wheel related graphs.

Definition 1.1. The degree of an edge $e=u v$ of $G$ is defined by $\operatorname{deg}(e)=\operatorname{deg}(u)+\operatorname{deg}(v)-2$ and it is equal to the number of edges adjacent to it. The maximum degree of an edge in $G$ is denoted by $\Delta^{\prime}(G)$.

Definition 1.2. The wheel graph $W_{n}$ is defined to be the join $K_{1}+C_{n}$. The vertex corresponding to $K_{1}$ is known as the apex and the vertices corresponding to cycle $C_{n}$ are known as rim vertices while the edges corresponding to cycle are known as rim edges.

Definition 1.3. The helm $H_{n}$ is the graph obtained from wheel $W_{n}$ by attaching a pendant edge to each rim vertex.

Definition 1.4. The closed helm $\mathrm{CH}_{n}$ is the graph obtained from helm $H_{n}$ by joining each pendant vertex to form a cycle.

Definition 1.5. The flower $F l_{n}$ is the graph obtained from helm $H_{n}$ by joining each pendant vertex to the apex of helm $H_{n}$.

Definition 1.6. The sunflower $S f_{n}$ is the graph obtained from flower $F l_{n}$ by attaching $n$ pendant edges to the apex of flower $F l_{n}$.

\section{Main Results}

Theorem 2.1. Let $G$ be any graph and $F$ be the restrained edge dominating set (REDS) of $G$ then edge domination number (EDN) of $G=$ restrained edge domination number (REDN) of $G$ if all the subgraphs of $E-F$ must be of minimum length two or $E(G) \neq F$.

Proof: Let $G=(V, E)$ be any graph and $F$ be the REDS of $G$. For a graph $G$, We assume that EDN=REDN.

To prove all the subgraphs of $E-F$ must be of minimum length two or $E(G) \neq F$. Suppose there is a subgraph of $E-F$ which is of length one. Since there is only one edge in $E-F$, by definition of restrained edge dominating set of $G, F$ can not be restrained edge dominating set but $F$ can be edge dominating set of $G$. It follows that $\mathrm{EDN} \neq \mathrm{REDN}$. Which is contradiction to our assumption that EDN=REDN. Which implies that all the subgraphs of $E-F$ must be of minimum length two.

Suppose $E(G)=F$. Consider $P_{4}$ with $E\left(P_{4}\right)=3$, for which $|F|=3$ as $\gamma_{r e}\left(P_{4}\right)=3$. In this case, $E-F=\phi$. Note that $\gamma_{e}\left(P_{4}\right)=1$, which implies that for $P_{4}$ EDN $\neq$ REDN. Which is contradiction to our assumption that EDN=REDN. So $E(G) \neq F$.

From above argument it is very clear to see that for graph $G, \mathrm{EDN}=\mathrm{REDN}$ if all the subgraphs of $E-F$ must be of minimum length two or $E(G) \neq F$.

Theorem 2.2. If $m$ is the number of independent edges of $G$ such that every independent edge must be in the restrained edge dominating $\operatorname{set}(\mathrm{REDS})$ of $G$ then $\gamma_{r e}(G) \geq m$.

Proof:Let $m \subseteq E(G)$ be the number of independent edges of $G$ and $F \subseteq E(G)$ be the REDS of $G$. Note that every independent edge of graph $G$ dominates its adjacent edges.

If every independent edge of $G$ is contained in $F$ and $|F|=|m|$ than clearly $\gamma_{r e}(G)=m$. If $F$ contains independent edges of $G$ and also the edges which are not independent then $|F|>|m|$, in this case $\gamma_{r e}(G)>m$. Thus, it is easy to see from both the cases that $\gamma_{r e}(G) \geq m$.

Theorem 2.3. For the helm $H_{n}, \gamma_{r e}\left(H_{n}\right)=\left\lceil\frac{n}{2}\right\rceil$.

Proof:Let $v_{1}, v_{2}, \ldots, v_{n}$ be the pendant vertices, $u_{1}, u_{2}, \ldots u_{n}$ be the rim vertices and $v$ be the apex of maximum degree $n$ of helm $H_{n}$. Let $e_{1}^{\prime}, e_{2}^{\prime}, \cdots, e_{n}^{\prime}$ be the pendant edges, $f_{1}, f_{2}, \ldots f_{n}$ be the rim edges and $e_{1}, e_{2}, \ldots, e_{n}$ be the internal edges of $H_{n}$. Note that $\left|V\left(H_{n}\right)\right|=2 n+1$ and $\left|E\left(H_{n}\right)\right|=3 n$.

We construct an edge set $F \subseteq E\left(H_{n}\right)$ as follows,

$F=\left\{e_{1}, e_{3}, e_{5}, \ldots, e_{2 i+1}\right\}$, where $0 \leq i \leq\left\lceil\frac{n}{2}\right\rceil-1$ with $|F|=\left\lceil\frac{n}{2}\right\rceil$. Since $F \subseteq E\left(H_{n}\right)$ and each edge in $E-F$ is adjacent to an edge in $F$ and another edge in $E-F$, the above set $F$ is a restrained edge dominating set of $\left(H_{n}\right)$. Moreover the above set $F$ is a minimal restrained edge dominating set 
of $\left(H_{n}\right)$ because for any edge $e \in F$, the set $F-\{e\}$ does not dominate the edges in $N(e)$ of $\left(H_{n}\right)$.

Now $\Delta^{\prime}\left(H_{n}\right)=n+2=\operatorname{deg}\left(e_{i}\right)$, for $1 \leq i \leq n$ and $\operatorname{deg}\left(f_{i}\right)=6$, for $1 \leq i \leq n$, also $\operatorname{deg}\left(e_{i}^{\prime}\right)=3$, for $1 \leq i \leq n$. So the edges in above set $F$ will dominate maximum number of distinct edges of $\left(H_{n}\right)$. Therefore any set containing the edges less than the number of edges in set $F$ will not dominate all the edges of $\left(H_{n}\right)$. This implies that above set $F$ is the restrained edge dominating set with minimum cardinality.

Hence set $F$ is a minimal restrained edge dominating set with minimum cardinality among all the minimal restrained edge dominating sets of $\left(H_{n}\right)$. Thus $\gamma_{r e}\left(H_{n}\right)=\left\lceil\frac{n}{2}\right\rceil$.

Illustration 2.4. The helm $H_{4}$ is shown in Figure 1 where the set of edges $\left\{e_{1}, e_{3}\right\}$ is its restrained edge dominating set of minimum cardinality.

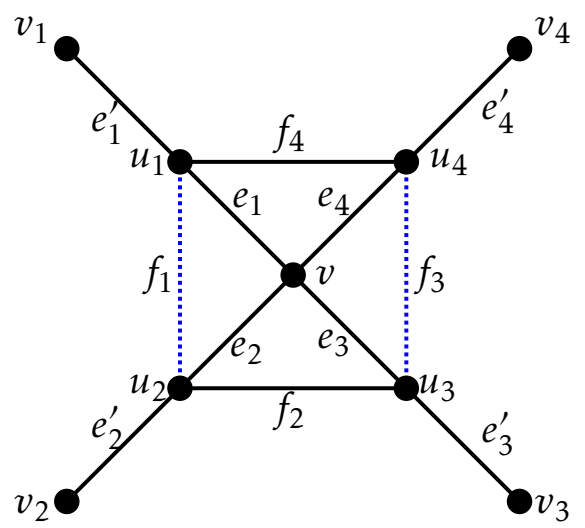

Figure 1: $\gamma_{r e}\left(H_{4}\right)=2$

Theorem 2.5. For the closed helm $\mathrm{CH}_{n}$, $\gamma_{r e}\left(C H_{n}\right)= \begin{cases}\left(\frac{2 n}{3}\right)+1 & ; \text { for } n \equiv 0(\bmod 3) \\ 2\left\lfloor\frac{n}{3}\right\rfloor+2 & ; \text { for } n \equiv 1 \text { or } 2(\bmod 3)\end{cases}$

Proof:The closed helm $\mathrm{CH}_{n}$ contains wheel $W_{n}$ and the outer cycle $C_{n}$. Let $V\left(\mathrm{CH}_{n}\right)=\left\{v, u_{1}, u_{2}, \cdots, u_{n}, v_{1}, v_{2}, \cdots, v_{n}\right\}$ and $E\left(C H_{n}\right)=\left\{e_{1}, e_{2}, \ldots, e_{n}, f_{1}, f_{2}, \ldots, f_{n}, e_{1}^{\prime}, e_{2}^{\prime}, \ldots, e_{n}^{\prime}, f_{1}^{\prime}, f_{2}^{\prime}\right.$, $\left.\ldots, f_{n}^{\prime}\right\}$. Note that $\left|V\left(C H_{n}\right)\right|=2 n+1$ and $\left|E\left(C H_{n}\right)\right|=4 n$.

Now we construct an edge set $F \subseteq E\left(C H_{n}\right)$ as follows.

$$
F=\left\{\begin{array}{c}
\left\{e_{1}\right\} \cup\left\{f_{3}, f_{6}, f_{9}, \ldots, f_{3 i}\right\} \cup\left\{f_{n-1}\right\} \cup\left\{f_{1}^{\prime}, f_{4}^{\prime}, \ldots, f_{3 i+1}^{\prime}\right\}, \\
\text { where } 0 \leq \mathrm{i} \leq\left(\frac{\mathrm{n}}{3}\right)-1, \text { for } \mathrm{n} \equiv(\bmod 3) \\
\left\{e_{1}\right\} \cup\left\{f_{3}, f_{6}, f_{9}, \ldots, f_{3 i}\right\} \cup\left\{f_{1}^{\prime}, f_{4}^{\prime}, \ldots, f_{3 i+1}^{\prime}\right\}, \\
\text { where } 0 \leq \mathrm{i} \leq\left\lfloor\frac{\mathrm{n}}{3}\right\rfloor, \text { forn } \equiv 1 \text { or } 2(\bmod 3)
\end{array}\right.
$$

with $|F|=\left(\frac{2 n}{3}\right)+1$, for $n \equiv 0(\bmod 3)$ and $|F|=2\left\lfloor\frac{n}{3}\right\rfloor+2$, for $n \equiv 1$ or $2(\bmod 3)$. Since $F \subseteq E\left(C H_{n}\right)$ and each edge in $E-F$ is adjacent to an edge in $F$ and another edge in $E-F$. The above set $F$ is a restrained edge dominating set of $\mathrm{CH}_{n}$. Moreover the set $F$ is a minimal restrained edge dominating set of $C H_{n}$ because for any edge $e \in F$, the set $F-\{e\}$ does not dominate the edges in $\mathrm{N}(e)$ of $\mathrm{CH}_{n}$.

Now $\operatorname{deg}\left(e_{i}\right)=n+2=\Delta^{\prime}\left(\mathrm{CH}_{n}\right)$, for $1 \leq i \leq n$ and $\operatorname{deg}\left(f_{i}\right)=6$, for $1 \leq i \leq n$ also $\operatorname{deg}\left(f_{i}^{\prime}\right)=4$, for $1 \leq i \leq n$. So the edges in the set $F$ will dominate maximum number of distinct edges of $\mathrm{CH}_{n}$. Therefore any set containing the edges less than the number of edges in the set $F$ will not dominate all the edges of $\mathrm{CH}_{n}$. This implies that above set $F$ is the restrained edge dominating set with minimum cardinality.

Hence set $F$ is a minimal restrained edge dominating set with minimum cardinality among all the minimal restrained edge dominating set of $\mathrm{CH}_{n}$. Thus

$$
\gamma_{r e}\left(C H_{n}\right)= \begin{cases}\left(\frac{2 n}{3}\right)+1 & ; \text { for } n \equiv 0(\bmod 3) \\ 2\left\lfloor\frac{n}{3}\right\rfloor+2 & ; \text { for } n \equiv 1 \text { or } 2(\bmod 3)\end{cases}
$$

Illustration 2.6. The closed helm $\mathrm{CH}_{4}$ is shown in Figure 2 where the set of edges $\left\{e_{1}, f_{3}, f_{1}^{\prime}, f_{4}^{\prime}\right\}$ is its restrained edge dominating set of minimum cardinality..

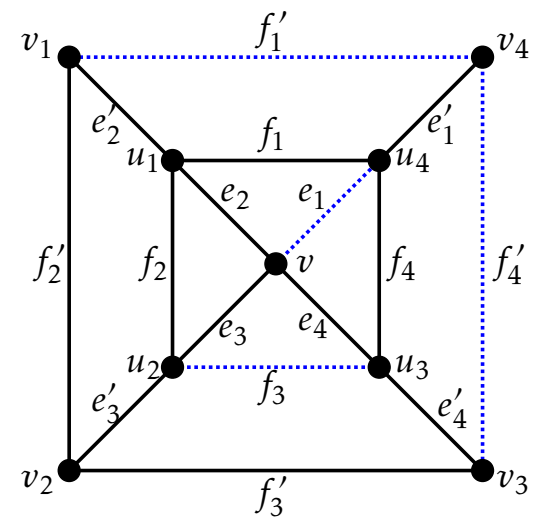

Figure 2: $\gamma_{r e}\left(\mathrm{CH}_{4}\right)=4$

Theorem 2.7. For the flower $F l_{n}$ and sunflower $S f_{n}$, $\gamma_{r e}\left(F l_{n}\right)=\left\lfloor\frac{n}{2}\right\rfloor+1=\gamma_{r e}\left(S f_{n}\right)$.

Proof: Let $V\left(F l_{n}\right)=\left\{v, u_{1}, u_{2}, \ldots, u_{n}, v_{1}, v_{2}, \ldots, v_{n}\right\}$ and $E\left(F l_{n}\right)=\left\{e_{1}, e_{2}, \ldots, e_{n}, f_{1}, f_{2}, \ldots, f_{n}, e_{1}^{\prime}, e_{2}^{\prime}, \ldots, e_{n}^{\prime}, e_{1}^{\prime \prime}, e_{2}^{\prime \prime}, \ldots, e_{n}^{\prime \prime}\right\}$. Note that $\left|V\left(F l_{n}\right)=2 n+1\right|$ and $\left|E\left(F l_{n}\right)\right|=4 n$.

Now, we construct an edge set $F \subseteq\left(F l_{n}\right)$ as follows. 
$F=\left\{e_{1}\right\} \cup\left\{f_{2}, f_{4}, f_{6}, \ldots, f_{2 i}\right\}$, where $1 \leq i \leq\left\lfloor\frac{n}{2}\right\rfloor$ with $|F|=\left\lfloor\frac{n}{2}\right\rfloor+1$. Since $F \subseteq E\left(F l_{n}\right)$ and each edge in $E-F$ is adjacent to an edge in $F$ and to another edge in $E-F$. The above set $F$ is a restrained edge dominating set of $F l_{n}$. Moreover the set $F$ is a minimal restrained edge dominating set of $F l_{n}$ because for any edge $e \in F$, the set $F-\{e\}$ does not dominate the edges in $N(e)$ of $F l_{n}$.

Now $\operatorname{deg}\left(e_{i}\right)=2 n+2=\Delta^{\prime}\left(F l_{n}\right)$, for $1 \leq i \leq n$ and $\operatorname{deg}\left(f_{i}\right)=6$, for $1 \leq i \leq n$. So the edges in the set $F$ will dominate maximum number of distinct edges of $F l_{n}$. Therefore any set containing the edges less than the number of edges in the set $F$ will not dominate all the edges of $F l_{n}$. This implies that above set $F$ is the restrained edge dominating set with minimum cardinality.

Hence set $F$ is a minimal restrained edge dominating set with minimum cardinality among all the minimal restrained edge dominating set of $F l_{n}$. For sunflower $S f_{n}$, it follows the same arguement with the same restrained edge dominating set $F$. Thus, $\gamma_{r e}\left(F l_{n}\right)=\left\lfloor\frac{n}{2}\right\rfloor+1=\gamma_{r e}\left(S f_{n}\right)$.

Illustration 2.8. The flower $\mathrm{Fl}_{4}$ is shown in Figure 3 where the set of edges $\left\{e_{1}, f_{2}, f_{4}\right\}$ is its restrained edge dominating set of minimum cardinality.

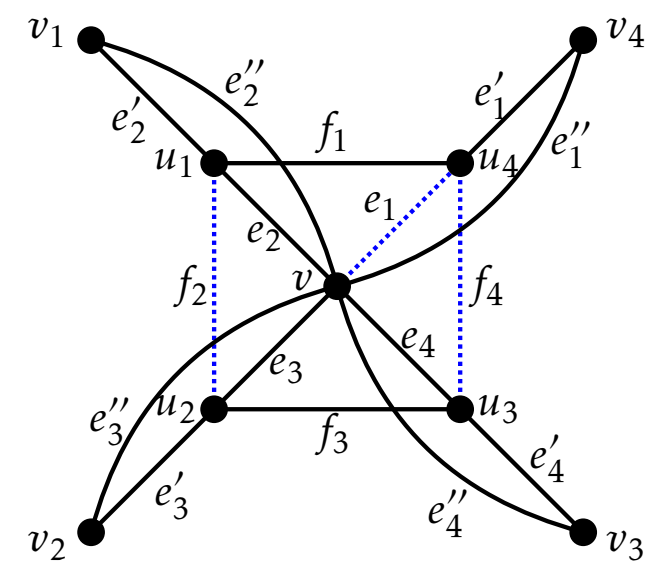

Figure 3: $\gamma_{r e}\left(F l_{4}\right)=3$

\section{Conclusion}

The concept of restrained edge domination is a variant of restrained domination and edge domination. To investigate the restrained edge domination number of graphs is always challenging and interesting. We have characterized restrained edge dominating set and also obtained the exact value of restrained edge domination number of helm $H_{n}$, closed helm $\mathrm{CH}_{n}$, flower graph $F l_{n}$ and sunflower graph $S f_{n}$. Similar results can be proved in the context of various types of dominating sets and different graph operations.

\section{Acknowledgment}

The authors are highly indebted to the anonymous referee for their kind comments and constructive suggestions on the first draft of this paper.

\section{References}

[1] S. Arumugam and S. Velammal, Edge Domination in Graphs, Taiwanese Journal of Mathematics, 2(1998), 173 179.

[2] G. S. Domke, J. H. Hattingh, S. T. Hedetiniemi, R. C. Laskar and L. R. Markus, Restrained domination in graphs, Discrete Mathematics, 203(1999), 61-69.

[3] G. S. Domke, J. H. Hattingh, M. A. Henning and L. R. Markus, Restrained Domination in Graphs with Minimum Degree Two, J. Combin. Math.Combin. Comput., 35(2000), 239-254.

[4] F. Harary, Graph Theory, Addison-Wesley, Reading, Mass, 1969.

[5] T. W. Haynes, S. T. Hedetniemi and P. J. Slater, Fundamentals of Domination in Graphs, Marcel Dekker, New York, 1998.

[6] V. R. Kulli and N. D. Soner, Complementary Edge Domination in Graphs, Indian Journal of Pure and Applied Mathematics, 28(1997), 917-920.

[7] S. Mitchell and S. Hedetniemi, Edge Domination in Trees, Congr. Numer, 19(1977), 489-509.

[8] N. D. Soner and S. Ghobadi, Restrained Edge Domination Number in Graphs, Adv. Stud. Contemp. Math., 19(2009), 143-149.

[9] J. A. Telle and A. Proskurowski, Algorithms for vertex partitioning problems on partial k-trees, SIAM J. Discrete Mathematics, 10(1997), 529-550.

${ }^{[10]}$ S. K. Vaidya and P. D. Ajani, Restrained Domination Number of Some Path Related Graphs, Journal of Computational Mathematica, 1(1) (2017), 114-121.

[11] S. K. Vaidya and P. D. Ajani, On Restrained Domination Number of Graphs, International Journal of Mathematics and Soft Computing, 8(1) (2018), 17-23.

[12] S. K. Vaidya and P. D. Ajani, On Restrained Domination Number of Some Wheel Related Graphs, Malaya Journal of Matematik, 7(1) (2019), 104-107.

[13] S. K. Vaidya and R. M. Pandit, Edge Domination in Some Path and Cycle Related Graphs, ISRN Discrete Mathematics, 2014(2014), 1-5.

[14] M. Yannakakis and F. Gavril, Edge Dominating Sets in Graphs, SIAM Journal on Applied Mathematics, 38(3)(1980), 364-372.

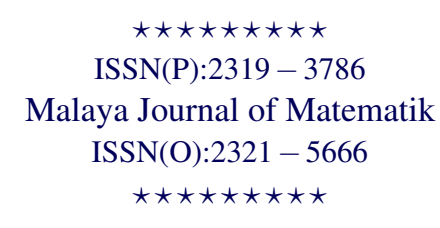

